\title{
EFFECTS OF TEMPERATURE DEPENDENT THERMAL CONDUCTIVITY ON NATURAL CONVECTION FLOW ALONG A VERTICAL FLAT PLATE WITH HEAT GENERATION
}

\author{
A. K. M. Safiqul Islam ${ }^{1}$, M. A. Alim², M. M. A. Sarker ${ }^{2}$, A. F. M. Khodadad Khan ${ }^{3}$
}

${ }^{1}$ Department of Mathematics, Gowripur Govt. College, Mymensingh, Email: safiqis@yahoo.com

${ }^{2}$ Department of Mathematics, Bangladesh University of Engineering and Technology, Dhaka-1000, Bangladesh, Email:maalim@math.buet.ac.bd, masarker@math.buet.ac.bd

${ }^{3}$ School of Engineering and Computer Science, Independent University Bangladesh, Email: khoda1936@gmail.com

\begin{abstract}
:
The effects of temperature dependent thermal conductivity on natural convection flow of an electrically conducting fluid along a vertical flat plate with heat generation have been investigated in this paper. The governing equations with associated boundary conditions for this phenomenon are converted to dimensionless forms using a suitable transformation. The transformed non-linear equations are then solved using the implicit finite difference method. Numerical results of the velocity and temperature profiles, skin friction coefficient and surface temperature profiles for different values of the thermal conductivity variation parameter, Prandtl number and heat generation parameters are presented graphically. Detailed discussion is given for the effects of the aforementioned parameters. Significant effect is found in the velocity and temperature profiles, skin friction coefficient and surface temperature distribution for the Prandtl number and heat generation parameters.
\end{abstract}

Keywords: thermal conductivity variation, heat generation, skin friction, Keller-box scheme.

\begin{tabular}{llll}
\hline NOMENCLATURE & $\bar{x}, \bar{y}$ & \multicolumn{1}{l}{ cartesian coordinates } \\
$b$ & plate thickness & $x, y$ & dimensionless cartesian coordinates \\
$C_{f x}$ & local skin friction coefficient & \multicolumn{2}{l}{ Greek symbols } \\
$c_{p}$ & specific heat at constant pressure & $\beta$ & co-efficient of thermal expansion \\
$f$ & dimensionless stream function & $\nabla$ & vector differential operator \\
$g$ & acceleration due to gravity & $\eta$ & dimensionless similarity variable \\
$G r$ & Grashof number & $\theta(x, 0)$ & surface temperature distribution \\
$h$ & dimensionless temperature & $\kappa_{f}$ & thermal conductivity of the fluid \\
$l$ & length of the plate & $\kappa_{s}$ & thermal conductivity of the solid \\
$Q$ & heat generation parameter & $\kappa_{\infty}$ & thermal conductivity of the ambient fluid \\
$P$ & conjugate conduction parameter & $\mu, v$ & dynamic and kinematic viscosities \\
$P r$ & Prandtl number & $\rho$ & density of the fluid \\
$T$ & temperature of the interface & $\sigma$ & electrical conductivity \\
$T_{b}$ & temperature at outside surface of the plate & $\tau_{w}$ & shearing stress \\
$T_{f}$ & temperature of the fluid & $\psi$ & stream function \\
$T_{\infty}$ & temperature of the ambient fluid & $\gamma$ & thermal conductivity variation parameter \\
$\bar{u}, \bar{v}$ & velocity components & &
\end{tabular}

\section{Introduction}

Electrically conducting fluid flow in presence of the effect of temperature dependent thermal conductivity and heat conduction problems are important from the technical point of view and such types of problems have 
received much attention by many researchers. In electronics in particular and in physics broadly used these terms. It is established that heat generation effects are generally rather more important both for gases and liquids.

Convection is the mode of energy transfer between a solid surface and the adjacent liquid or gas that is in motion and it involves the combined effects of conduction and fluid motion. The faster the fluid motion, the greater the heat transfer due to convection.

A medium through which heat is conducted may involve the conversation of electrical, nuclear or chemical energy into heat (or thermal) energy. In heat conduction analysis, such conversion processes are characterized as heat generation. For example, the temperature of a resistance wire rises rapidly when electric current passes through it, as a result of the electrical energy are converted to heat at a rate of $I^{2} R$, where $I$ is the current and $R$ is the electrical resistance of the wire. The safe and effective removal of this heat away from the sites of heat generation (the electronic circuits) is the subject of electronics cooling which is one of the modern application areas of heat transfer. Likewise, a large amount of heat is generated in the fuel elements of nuclear reactors as a result of nuclear fission that serves as the heat source for the nuclear power plants. The heat generated in the Sun as a result of the fusion of hydrogen into helium makes the Sun a large nuclear reactor that supplies heat to the earth. Another source of heat generation in a medium is exothermic chemical reactions that may occur throughout the medium. The chemical reaction in this case serves as a heat source for the medium. In the case of endothermic reactions, however, heat is absorbed instead of being released during reaction and thus the chemical reaction serves as a heat sink. The heat generation term becomes a negative quantity in this case. Heat generation is a volumetric phenomenon. That is, it occurs throughout the body of a medium. Therefore, the rate of heat generation in a medium is usually specified per unit volume. Heat generation is the ability to emit greater-than-normal heat from the body.

Model studies of the free and mixed convection flows have earned reputations because of their applications in geophysical, geothermal and nuclear engineering problems. Also the problems of various types of shapes over or on a free convection boundary layer flow have been studied by many researchers. Amongst them Miyamoto et al. (1980) studied the effect of axial heat conduction in a vertical flat plate on free convection heat transfer. Pozzi and Lupo (1988) investigated the coupling of conduction with laminar convection along a flat plate. Pop et al. (1995) investigated the conjugate mixed convection on a vertical surface in porous medium. Gebhart (1962) investigated the effect of dissipation natural convection. Takhar and Soundalgekar (1980) studied the dissipation effects on MHD free convection flow past a semi-infinite vertical plate. Khan (2002) investigated the conjugate effect of conduction and convection with natural convection flow from a vertical flat plate. Mamun (2005) studied the effects of conduction and convection on magnetohydrodynamic flow with and without viscous dissipation from a vertical flat plate. Hossain (1992) analyzed the viscous and Joule heating effects on MHD free convection flow with variable plate temperature. Rahman et al. (2008) investigated the effects of temperature dependent thermal conductivity on MHD free convection flow along a vertical flat plate with heat conduction. Rahman and Alim (2009) analyzed numerical study of MHD free convective heat transfer flow along a vertical flat plate with temperature dependent thermal conductivity. Nasrin and Alim (2009) studied the combined effects of viscous dissipation and temperature dependent thermal conductivity on MHD free convection flow with conduction and joule heating along a vertical flat plate. Alim et al. (2008) analyzed the combined effect of viscous dissipation $\&$ joule heating on the coupling of conduction $\&$ free convection along a vertical flat plate. Alim et al. (2007) investigated joule heating effect on the coupling of conduction with MHD free convection flow from a vertical flat plate. Chowdhury and Islam (2000) analyzed MHD Free Convection Flow of Visco-elastic Fluid past an Infinite Porous Plate. Alam et al. (2007) discussed free convection from a vertical permeable circular cone with pressure work and non-uniform surface temperature. Miraj et al. (2010) studied effect of radiation on natural convection flow on a sphere in presence of heat generation. Ali et al. (2011) investigated Conjugate effects of Radiation and Joule Heating on Magnetohydrodynamic Free Convection Flow along a sphere with Heat Generation. Miraj et al. (2011) analyzed effects of pressure work and on natural convection flow around a sphere with heat generation.

The present study is to incorporate the idea of the effects of temperature dependent thermal conductivity on natural convection boundary layer flow along a vertical flat plate with heat generation. The governing boundary layer equations are transformed into a non-dimensional form and the resulting non-linear system of partial differential equations is reduced to local non-similar partial differential forms by adopting appropriate transformations. The transformed boundary layer equations are then solved numerically. Numerical results of the velocity and temperature profiles, local skin friction coefficient and surface temperature profiles for the 
thermal conductivity variation parameter, Prandtl number and heat generation parameters are presented graphically.

\section{Mathematical Formulation of the Problem}

At first we consider a steady two-dimensional laminar natural convection flow of an electrically conducting, viscous and incompressible fluid along a vertical flat plate of length $l$ and thickness $\mathrm{b}$ (Fig.-1). It is assumed that the temperature at the outer surface of the plate is maintained at a constant temperature $T_{b}$, where $T_{b}>T_{\infty}$, the ambient temperature of the fluid. The $\bar{y}$-axis i.e. normal direction to the surface and $\bar{x}$-axis is taken along the flat plate. The coordinate system and the configuration are shown in Figure 1.

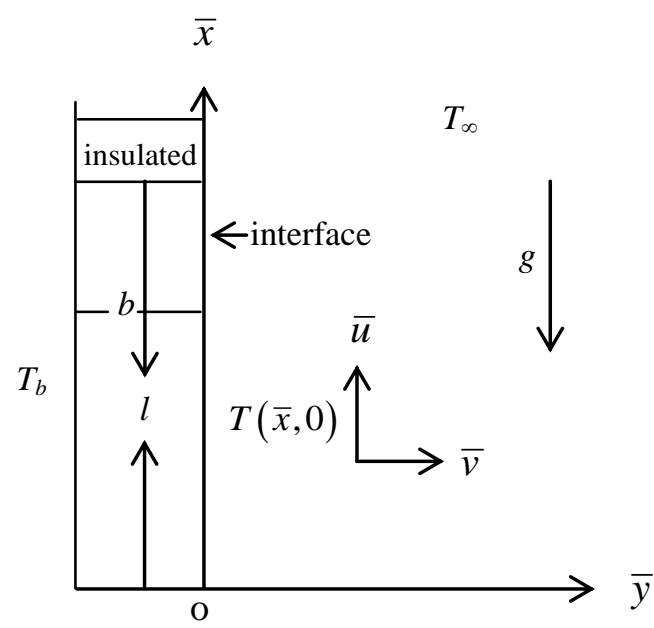

Figure.1: Physical model and coordinate system

The governing equations of such laminar flow with heat generation and thermal conductivity variation along a vertical flat plate under the Boussinesq approximations $\rho=\rho_{\infty}\left[1-\beta\left(T_{b}-T_{\infty}\right)\right]$, where $\rho_{\infty}$ and $T_{\infty}$ are the density and temperature respectively outside the boundary layer are given below. For the present problem the continuity, momentum and energy equations take the following form

$$
\begin{aligned}
& \frac{\partial \bar{u}}{\partial \bar{x}}+\frac{\partial \bar{v}}{\partial \bar{y}}=0 \\
& \bar{u} \frac{\partial \bar{u}}{\partial \bar{x}}+\bar{v} \frac{\partial \bar{u}}{\partial \bar{y}}=v \frac{\partial^{2} \bar{u}}{\partial \bar{y}^{2}}+g \beta\left(T_{f}-T_{\infty}\right) \\
& \bar{u} \frac{\partial T_{f}}{\partial \bar{x}}+\bar{v} \frac{\partial T_{f}}{\partial \bar{y}}=\frac{1}{\rho C_{p}} \frac{\partial}{\partial \bar{y}}\left(\kappa_{f} \frac{\partial T_{f}}{\partial \bar{y}}\right)+\frac{Q_{0}}{\rho C_{p}}\left(T_{f}-T_{\infty}\right)
\end{aligned}
$$

Here $\beta$ is coefficient of volume expansion. The temperature dependent thermal conductivity, which is used by Rahman (2008) as follows

$$
\kappa_{f}=\kappa_{\infty}\left[1+\delta\left(T_{f}-T_{\infty}\right)\right]
$$

Where $\kappa_{\infty}$ is the thermal conductivity of the ambient fluid and $\delta$ is a constant, defined as $\delta=\frac{1}{\kappa_{f}}\left(\frac{\partial \kappa}{\partial T}\right)_{f}$. The appropriate boundary condition is to be satisfied by the above equations are

$$
\begin{aligned}
& \bar{u}=0, \bar{v}=0 \\
& \left.T_{f}=T(\bar{x}, 0), \frac{\partial T_{f}}{\partial \bar{y}}=\frac{\kappa_{s}}{b \kappa_{f}}\left(T_{f}-T_{b}\right)\right\} \text { on } \bar{y}=0, \bar{x}>0 \\
& \bar{u} \rightarrow 0, T_{f} \rightarrow T_{\infty} \text { as } \bar{y} \rightarrow \infty, \bar{x}>0
\end{aligned}
$$


The non-dimensional governing equations and boundary conditions can be obtained from Equations (1) - (3) using the following dimensionless quantities

$$
\begin{aligned}
& x=\frac{\bar{x}}{l}, \quad y=\frac{\bar{y}}{l} G r^{\frac{1}{4}}, u=\frac{\bar{u} l}{v} G r^{-\frac{1}{2}}, v=\frac{\bar{v} l}{v} G r^{-\frac{1}{4}}, \\
& \theta=\frac{T_{f}-T_{\infty}}{T_{b}-T_{\infty}}, G r=\frac{g \beta l^{3}\left(T_{b}-T_{\infty}\right)}{v^{2}}
\end{aligned}
$$

where $l$ is the length of the plate, $G r$ is the Grashof number, $\theta$ is the dimensionless temperature.

Now from Equations (1)-(3), we get using the following dimensionless equations

$$
\frac{\partial u}{\partial x}+\frac{\partial u}{\partial y}=0
$$

$u \frac{\partial u}{\partial x}+v \frac{\partial u}{\partial y}=\frac{\partial^{2} u}{\partial y^{2}}+\theta$

$u \frac{\partial \theta}{\partial x}+v \frac{\partial \theta}{\partial y}=\frac{1}{\operatorname{Pr}}(1+\gamma \theta) \frac{\partial^{2} \theta}{\partial y^{2}}+\frac{\gamma}{\operatorname{Pr}}\left(\frac{\partial \theta}{\partial y}\right)^{2}+Q \theta$

where, $\operatorname{Pr}=\frac{\mu C_{p}}{\kappa}$ is the Prandtl number, $\gamma=\delta\left(T_{b}-T_{\infty}\right)$ is the non-dimensional thermal conductivity variation parameter and $Q=\frac{Q_{0} l^{2}}{\mu C_{p} G r^{1 / 2}} \quad$ is the dimensionless heat generation parameter. The corresponding boundary conditions (5) then take the following form

$u=0, v=0, \theta-1=\left(\begin{array}{ll}1+\gamma & \theta\end{array}\right) \frac{\partial \theta}{\partial y}$ on $y=0, x>0$

$u \rightarrow 0, \theta \rightarrow 0$ as $y \rightarrow \infty, x>0$

where, $p=\left(\frac{\kappa_{\infty} b}{\kappa_{s} l}\right) G r^{1 / 4}$ is the conjugate conduction parameter. The described problem is governed by the coupling parameter $\mathrm{p}$. In actual fact, magnitude of $O(p)$ depends on $b / l$ and $G r^{1 / 4}$ being the order of unity. Since $l$ is small, the term $b / l$ becomes greater than one. For air, $\frac{\kappa_{\infty}}{\kappa_{s}}$ attains very small values if the plate is highly conductive and reaches the order of 0.1 for materials such as glass. Therefore in different cases $p$ is different but not always a small number. In the present investigation we have considered $p=1$ which is accepted for $b / l$ of $O\left(\frac{\kappa_{\infty}}{\kappa_{s}}\right)$.

To solve Equations (8) and (9) subject to the boundary conditions (10) the following transformations are proposed by Merkin \& Pop (1996)

$$
\begin{aligned}
& \psi=x^{\frac{4}{5}}(1+x)^{\frac{1}{-20}} f(x, \eta), \\
& \eta=y \quad x^{-\frac{1}{5}}(1+x)^{-\frac{1}{20}}, \\
& \theta=x^{\frac{1}{5}}(1+x)^{-\frac{1}{5}} h \quad(x, \eta)
\end{aligned}
$$

here $\eta$ is the similarity variable and $\psi$ is the non-dimensional stream function which satisfies the continuity equation and is related to the velocity components in the usual way as

$$
u=\frac{\partial \psi}{\partial y} \text { and } v=-\frac{\partial \psi}{\partial x} .
$$


Moreover, $h(x, \eta)$ represents the non-dimensional temperature. The momentum and energy equations are transformed for the new co-ordinate system. At first, the velocity components are expressed in terms of the new variables for this transformation. Thus the following equations

$$
\begin{aligned}
& f^{\prime \prime \prime}+\frac{16+15 x}{20(1+x)} f f^{\prime \prime}-\frac{6+5 x}{10(1+x)} f^{\prime 2}+h=x\left(f^{\prime} \frac{\partial f^{\prime}}{\partial x}-f^{\prime \prime} \frac{\partial f}{\partial x}\right) \\
& \frac{1}{\operatorname{Pr}} h^{\prime \prime}+\frac{\gamma}{\operatorname{Pr}}\left(\frac{x}{1+x}\right)^{\frac{1}{5}} h h^{\prime \prime}+\frac{\gamma}{\operatorname{Pr}}\left(\frac{x}{1+x}\right)^{\frac{1}{5}} h^{\prime 2}+\frac{16+15 x}{20(1+x)} f h^{\prime}+ \\
& Q x^{2 / 3}(1+x)^{1 / 10} h-\frac{1}{5(1+x)} f^{\prime} h=x\left(f^{\prime} \frac{\partial h}{\partial x}-h^{\prime} \frac{\partial f}{\partial x}\right)
\end{aligned}
$$

where prime denotes partial differentiation with respect to $\eta$. The boundary conditions as mentioned in Equation (10) then take the following form

$$
\begin{aligned}
& f(x, 0)=f^{\prime}(x, 0)=0 \\
& h^{\prime}(x, 0)=\frac{x^{\frac{1}{5}}(1+x)^{-\frac{1}{5}} h(x, 0)-1}{(1+x)^{-\frac{1}{4}}+\gamma x^{\frac{1}{5}}(1+x)^{-\frac{9}{20}} h(x, 0)} \\
& f^{\prime}(x, \infty) \rightarrow 0, h(x, \infty) \rightarrow 0
\end{aligned}
$$

The set of Equations (12) and (13) together with the boundary conditions (14) are solved by applying implicit finite difference method with Keller-box elimination technique (1978) scheme. From the process of numerical computation, in practical point of view, it is important to calculate the values of the surface shear stress in terms of the skin friction coefficient. This can be written in the non-dimensional form as Molla et al. (2005)

$$
C_{f}=\frac{G r^{-\frac{3}{4}} l^{2}}{\mu v} \tau_{w}
$$

where $\tau_{w}\left[=\mu\left(\begin{array}{lll}\partial & \bar{u} / \partial & \bar{y}\end{array}\right)_{\bar{y}=0}\right]$ is the shearing stress. Using the new variables described in (6), the local skin friction co-efficient can be written as

$$
C_{f x}=x^{\frac{2}{5}}(1+x)^{-\frac{3}{20}} f^{\prime \prime}(x, 0)
$$

In practical point of view, it is important to calculate the values of the surface temperature. The numerical values of the surface temperature are obtained from the relation. This can written in the non-dimensional form as

$$
\theta(x, 0)=x^{\frac{1}{5}}(1+x)^{-\frac{1}{5}} h(x, 0)
$$

We have also discussed the relations for velocity and the temperature profiles for different values of the thermal conductivity variation parameter, Prandtl number and heat generation parameter.

\section{Numerical Method of Solution}

The numerical method used is finite difference method together with Keller- box Scheme (1978). To begin with the partial differential eqs. (12)- (13) are first converted into a system of first order differential equations. Then these equations are expressed in finite difference forms by approximating the functions and their derivatives in terms of the center difference. Denoting the mesh points in the $x, \eta$-plane by $x_{\mathrm{i}}$ and $\eta_{\mathrm{j}}$ where $\mathrm{i}=1,2, \ldots, \mathrm{M}$ and $\mathrm{j}$ $=1,2, \ldots, \mathrm{N}$, central difference approximations are made, such that those equations involving $x$ explicitly are centered at $\left(x_{\mathrm{i}-1 / 2}, \eta_{\mathrm{j}-1 / 2}\right)$ and the remainder at $\left(x_{\mathrm{i}}, \eta_{\mathrm{j}-1 / 2}\right)$, where $\eta_{j-1 / 2}=\frac{1}{2}\left(\eta_{j}+\eta_{j-1}\right)$ etc. The above central difference approximations reduces the system of first order differential equations to a set of non-linear difference equations for the unknown at $x_{\mathrm{i}}$ in terms of their values at $x_{\mathrm{i}-1}$. The resulting set of nonlinear difference equations are solved by using the Newton's quasi-linearization method. The Jacobian matrix has a block-tridiagonal structure and the difference equations are solved using a block-matrix version of the Thomas 
algorithm; the details of the computational procedure have been discussed further by in the book by Cebecci and Bradshow (1984) and widely used by Hossain (1992) and Hossain et al. (1999).

\section{Validity of the Present Work}

Table 1 and 2 depict the comparisons of the present numerical results of the skin friction coefficient $C_{f x}$ and the surface temperature $\theta(x, 0)$ with those obtained by Pozzi and Lopo (1988) and Merkin and Pop (1996) respectively. Here, the thermal conductivity variation parameter $\gamma$ and heat generation parameter $Q$ are ignored (i.e. $\gamma=0.0$ and $Q=0.0$ ) and the Prandtl number $\operatorname{Pr}=0.733$ with $x^{\frac{1}{5}}=\xi$ is chosen. It is clearly seen that there is an excellent agreement among the present results with the solutions Pozzi and Lopo (1988) and Merkin and Pop (1996).

Table 1: Comparison of the present numerical results of skin friction coefficient $C_{f x}$ with Prandtl number $\operatorname{Pr}=$ $0.733, \gamma=0.0$ and $Q=0.0$ against $x$.

\begin{tabular}{|c|c|c|c|}
\hline \multicolumn{3}{|c|}{$C_{f x}$} \\
\hline$x^{\frac{1}{5}}=\xi$ & Pozzi and Lupo (1988) & Merkin and Pop (1996) & Present work \\
\hline 0.7 & & & 0.423 \\
\hline 0.8 & 0.430 & 0.430 & 0.528 \\
\hline 0.9 & 0.530 & 0.530 & 0.633 \\
\hline 1.0 & 0.635 & 0.635 & 0.748 \\
\hline 1.1 & 0.741 & 0.745 & 0.857 \\
\hline 1.2 & 0.829 & 0.859 & 0.972 \\
\hline
\end{tabular}

Table 2: Comparison of the present numerical results of surface temperature $\theta(x, 0)$ with Prandtl number $P r=$ $0.733, \gamma=0.0$ and $Q=0.0$ against $x$.

\begin{tabular}{|c|c|c|c|}
\hline \multicolumn{3}{|c|}{$\theta(x, 0)$} \\
\hline$x^{\frac{1}{5}}=\xi$ & Pozzi and Lupo (1988) & Merkin and Pop (1996) & Present work \\
\hline 0.7 & 0.651 & 0.651 & 0.651 \\
\hline 0.8 & 0.684 & 0.686 & 0.687 \\
\hline 0.9 & 0.708 & 0.715 & 0.716 \\
\hline 1.0 & 0.717 & 0.741 & 0.742 \\
\hline 1.1 & 0.699 & 0.762 & 0.763 \\
\hline 1.2 & 0.640 & 0.781 & 0.781 \\
\hline
\end{tabular}

\section{Results and Discussion}

The objective of the present work is to analyze the effect of temperature dependent thermal conductivity on electrically conducting fluid on free convection flow along a vertical flat plate with heat generation. In this simulation the values of the Prandtl number $\mathrm{Pr}$ are considered to be $0.73,1.00,1.50,2.00$ and 2.50 that corresponds to hydrogen, steam, sulfur dioxide, ammonia and methyl chloride respectively. Detailed numerical results of the velocity, temperature, skin friction coefficient and surface temperature profiles obtained for different values of the thermal conductivity variation parameter, Prandtl number and heat generation parameter are presented graphically. Numerical computation are carried out for a range of thermal conductivity variation parameter $\gamma=0.01,0.12,0.25,0.36,0.50$ and heat generation parameter $Q=0.01,0.12,0.25,0.36,0.50$.

The velocity and the temperature fields obtained from the solutions of Equations (12) to (13) and the local skin friction coefficient and surface temperature distribution fields obtained from the solutions of Equations (16) to (17) are depicted in Figures 2 to 7. 
The effect of thermal conductivity variation parameter $\gamma$ on the velocity and temperature profiles against $\eta$ within the boundary layer with $\operatorname{Pr}=0.73$ and $Q=0.01$ are shown in Figures 2(a) and 2(b), respectively. It is seen from Figures 2(a) and 2(b) that the velocity and temperature increase within the boundary layer with the increasing values of $\gamma$. It means that the velocity boundary layer and the thermal boundary layer thickness expand for large values of $\gamma$. But the effects are small in both cases.

Figures 3(a) and 3(b) illustrate the velocity and temperature profiles against $\eta$ for different values of Prandtl number $\operatorname{Pr}$ with $\gamma=0.01$ and $Q=0.01$. From Figure 3(a), it can be observed that the velocity decreases as well as its position moves toward the interface with the increasing values of $P r$. From Figure 3(b), it is seen that the temperature profiles shift downward with the increasing $P r$. Prandtl number $P r$ is the ratio of momentum diffusivity (kinematic viscosity) to thermal diffusivity. Prandtl number $P r$ increases means that kinematic viscosity increases. For this reason, velocity decreases. When thermal diffusivity decreases, Prandtl number $\mathrm{Pr}$ increases for constant kinematic viscosity. Then we see that temperature decreases. Significant effect is found both these cases.
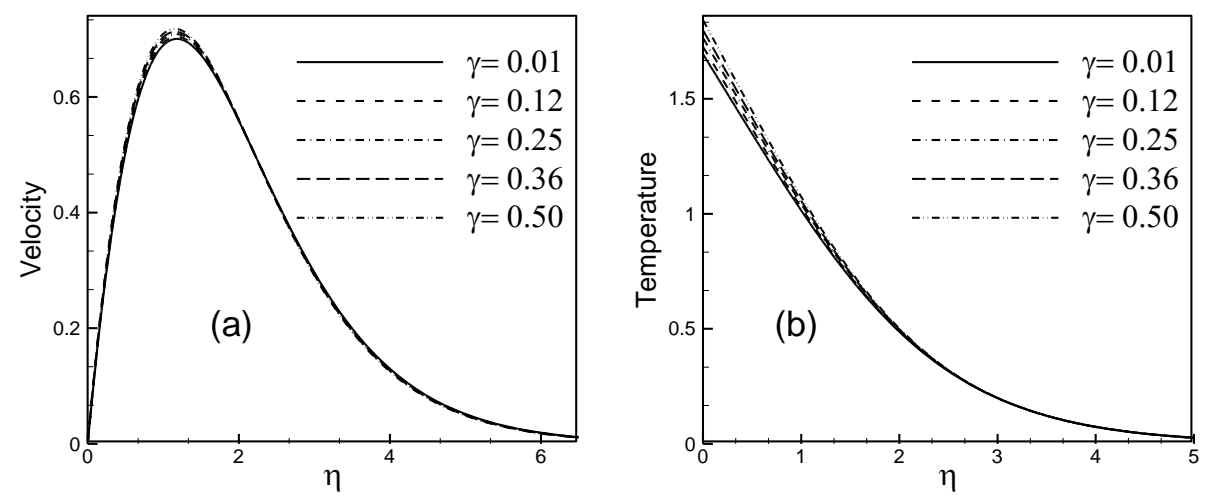

Figure 2: (a) Velocity and (b) Temperature profiles against $\eta$ for different values of $\gamma$ with $\operatorname{Pr}=0.73$ and $Q=$ 0.01 .
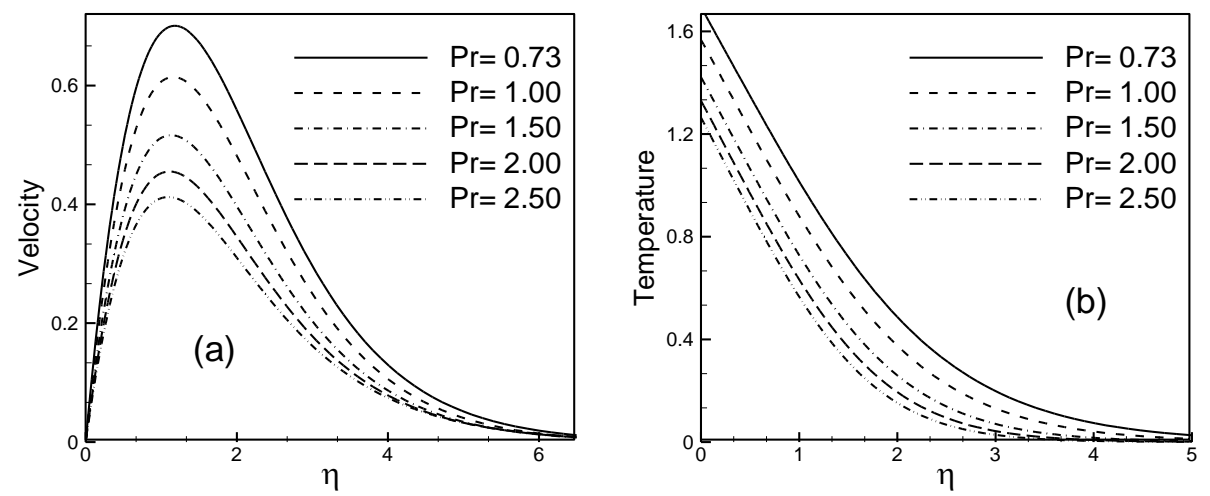

Figure 3: (a) Velocity and (b) Temperature profiles against $\eta$ for different values of $\operatorname{Pr}$ with $\gamma=0.01$ and $Q=$ 0.01 .

In Figures 4(a) and 4(b) describe the velocity and temperature profiles against $\eta$ for different values of heat generation parameter $Q$ with $\gamma=0.01$ and $\operatorname{Pr}=0.73$. From Figure 4(a), it can be observed that the velocity increases as well as its position moves upward the interface with the increasing values of $Q$. From Figure 4(b), it is seen that the temperature profiles also the same as increasing within the boundary layer. It means that the velocity boundary layer and the thermal boundary layer thickness expand for large values of $Q$.

Figures 5(a) and 5(b) illustrate the effect of the thermal conductivity variation parameter $\gamma$ on the skin friction coefficient $C_{f x}$ and surface temperature $\theta(x, 0)$ against $x$ with $\operatorname{Pr}=0.73$ and $Q=0.01$. It is seen that the skin friction increases monotonically along the upward direction of the plate for a particular values of $\gamma$. It is also seen that that the skin friction increases for the increasing values of $\gamma$. The same result is observed for the surface temperature from Figure 5(b). This is to be expected because the higher value for the thermal conductivity 
variation parameter accelerates the fluid flow and increases the velocity and temperature as mentioned in Figures 2(a) and 2(b) respectively.
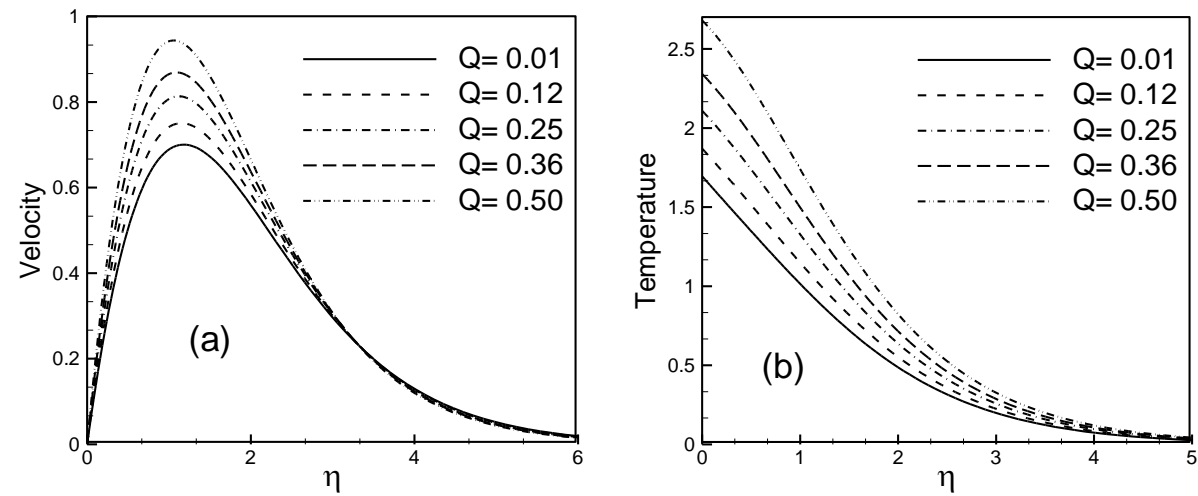

Figure 4: (a) Velocity and (b) Temperature profiles against $\eta$ for different values of $Q$ with $\gamma=0.01$ and $\operatorname{Pr}=$ 0.73 .
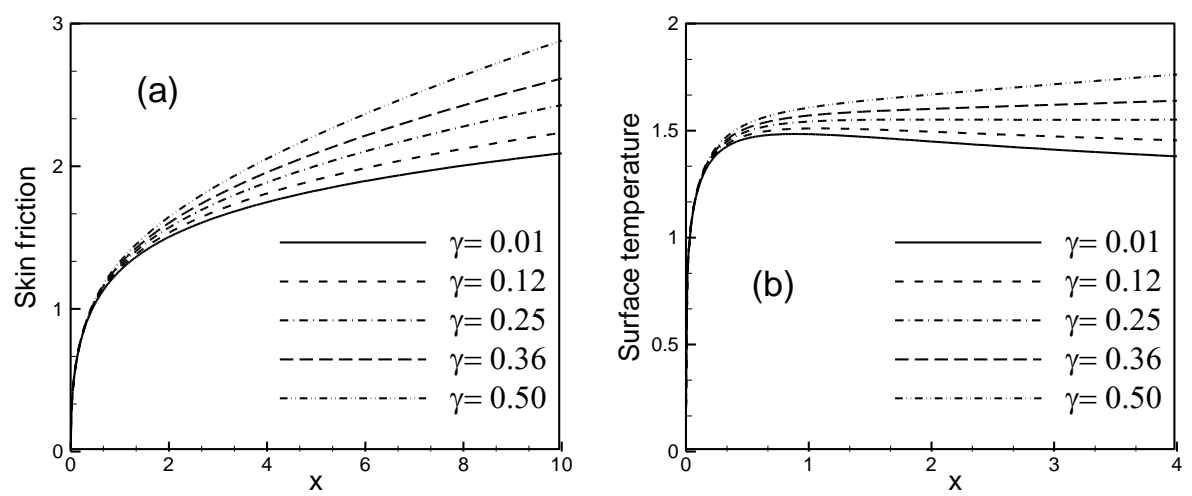

Figure 5: (a) Local skin friction coefficient $C_{f x}$ and (b) Surface temperature distribution $\theta(x, 0)$ against $x$ for different values of $\gamma$ with $\operatorname{Pr}=0.73$ and $Q=0.01$.
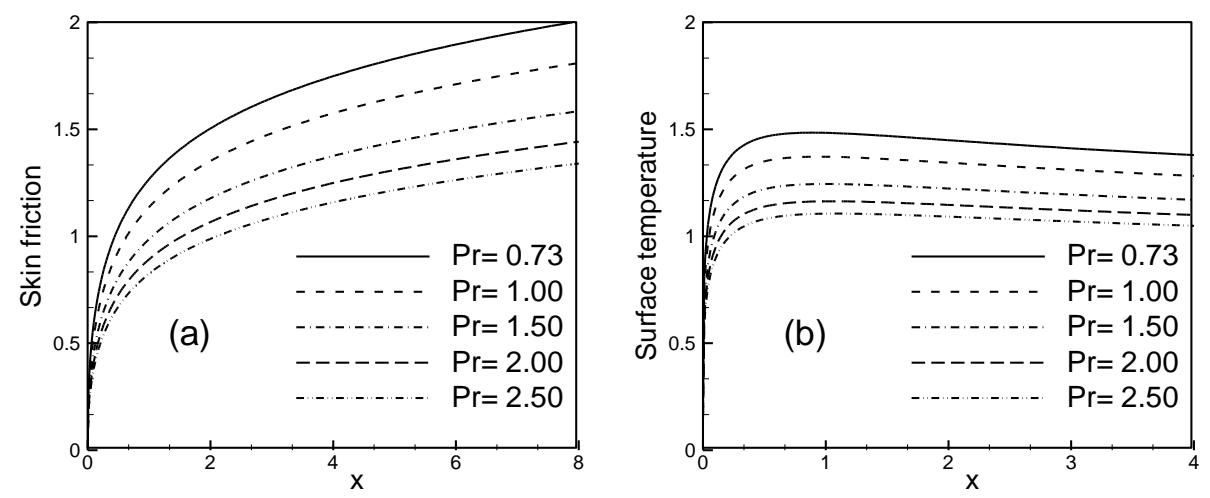

Figure 6: (a) Local skin friction coefficient $C_{f x}$ and (b) Surface temperature distribution $\theta(x, 0)$ against $x$ for different values of $\operatorname{Pr}$ with $\gamma=0.01$ and $Q=0.01$.

Figures 6(a) and 6(b) deal with the effect of Prandtl number $P r$ on the local skin friction $C_{f x}$ and surface temperature distribution $\theta(x, 0)$ against $x$ with $\gamma=0.01$ and $Q=0.01$. It can be observed from Figure 6(a) that the skin friction increases monotonically for a particular value of $P r$. It can also be noted that the skin friction coefficient decreases for the increasing values of $P r$. From Figure 6(b), it can be seen that the surface temperature decreases when $P r$ increases along the positive $x$ direction for a particular value of $P r$. This is 
expected because when Prandtl number $P r$ increases the skin friction coefficient and the surface temperature both decrease due to viscosity.

From Figures 7(a) and 7(b) we see the effect of $Q$ on the skin friction coefficient $C_{f x}$ and surface temperature $\theta(x, 0)$ against $x$ with $\gamma=0.01$ and $\operatorname{Pr}=0.73$. It can be noted that the skin friction and surface temperature profile increase for the increasing values of $Q$. When internal heat is generated the skin friction and surface temperature also are increased. Significant effect is found both these cases.
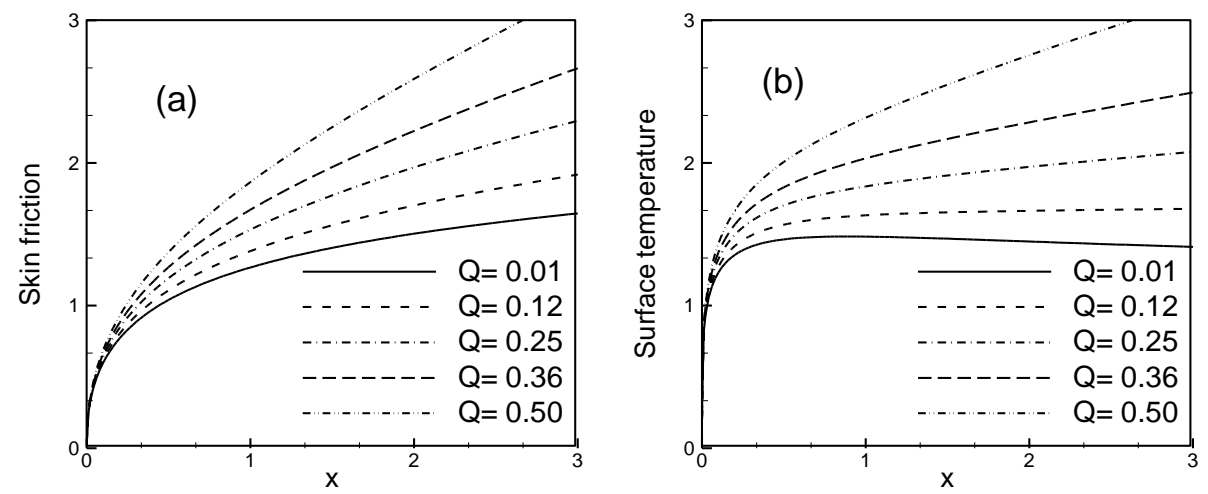

Figure 7: (a) Local skin friction coefficient $C_{f x}$ and (b) Surface temperature distribution $\theta(x, 0)$ against $x$ for different values of $Q$ with $\gamma=0.01$ and $\operatorname{Pr}=0.73$.

\section{Conclusion}

The effects of temperature dependent thermal conductivity on free convection flow along a vertical flat plate with heat generation have been presented graphically. From this investigation the following conclusions may be drawn:

- The velocity within the boundary layer increases for decreasing values of Pr and for increasing values of $\gamma$ and $\mathrm{Q}$.

- The temperature within the boundary layer increases for increasing values of $\gamma$ and Q and for decreasing values of Pr.

- The local skin friction coefficient decreases for the increasing values of Pr and increases for increasing values of $\gamma$ and $Q$.

- An increase in the values of $\gamma$ and Q leads to an increase in surface temperature. On the other hand, this decreases for increasing values of $\mathrm{Pr}$.

- For increased value of the thermal conductivity variation parameter, the velocity and the temperature profiles increase slightly.

- Significant effects are found in skin friction and surface temperature for the Prandtl number and heat generation parameters.

- The local skin friction coefficient and surface temperature distribution are more clear than the velocity and temperature profiles.

\section{Reference}

Miyamoto, M., Sumikawa, J. Akiyoshi, T. and Nakamura, T., (1980), The effect of axial heat conduction in a vertical flat plate on free convection heat transfer, Int. J. Heat Mass Transfer, Vol. 23, No. 11, pp.1545-1553.

Pozzi, A. and Lupo, M., (1988), The coupling of conduction with laminar convection along a flat plate. Int. J. Heat Mass Transfer, Vol. 31, No. 9, pp.1807-1814. doi:10.1016/0017-9310(88)90195-0

Pop, I. Lesnic, D. and Ingham, D. B., (1995), The conjugate mixed convection on a vertical surface in porous medium, Int. J. Heat transfer, Vol. 38, No. 8 pp.1517-1525. 
Gebhart, B., (1962), Effect of dissipation on natural convection, J. Fluid Mechanics, Vol. 14, No. 2 , pp. 225 232.

Takhar, H. S. and Soundalgekar, V. M., (1980), Dissipation effects on MHD free convection flow past a semiinfinite vertical plate, Applied Scientific Research, Vol. 36, No. 3, pp. 163-171.

Khan, Z. I., (2002), Conjugate effect of conduction and convection with natural convection flow from a vertical flat plate and in an inclined square cavity, M. Phil thesis, Department of Mathematics, BUET.

Mamun, A. A., (2005), Effects of conduction and convection on magnetohydrodynamic flow with and without dissipation from a vertical flat plate. M. Phil thesis, Department of Mathematics, BUET.

Hossain, M. A., (1992), Viscous and Joule heating effects on MHD free convection flow with variable plate temperature, Int. J. Heat Mass transfer, Vol. 35, No. 12, pp.3485-3487. doi:10.1016/0017-9310(92)90234-J

Rahman, M. M, Mamun A. A, Azim M. A and Alim, M. A., (2008), Effects of temperature dependent thermal conductivity on magnetohydrodynamic (MHD) free convection flow along a vertical flat plate with heat conduction, Nonlinear Analysis: Modeling and Control, Vol.13, No. 4,pp. 513-524.

Rahman, M. M and Alim, M. A., (2009), Numerical study of magnetohydrodynamic (MHD) free convective heat transfer flow along a vertical flat plate with temperature dependent thermal conductivity, Journal of Naval Architecture and Marine Engineering, JNAME, Vol.6, No.1, pp.16-29, doi: 10.3329/jname.v6i1.2654

Nasrin, R. and Alim, M. A., (2009), Combined effects of viscous dissipation and temperature dependent thermal conductivity on magnetohydrodynamic (MHD) free convection flow with conduction and joule heating along a vertical flat plate, Journal of Naval Architecture and Marine Engineering, JNAME, Vol.6, No.1, pp.30-40, doi: 10.3329/jname.v6i1.2648.

Alim, M. A., Alam, Md. M., Mamun A.A. and Hossain, Md. B., (2008), Combined effect of viscous dissipation $\&$ joule heating on the coupling of conduction \& free convection along a vertical flat plate, Int. Communications in Heat \& Mass Transfer, Vol. 35, No.3, pp. 338-346.

Alim, M. A., Alam, M. and Mamun, A. A., (2007), Joule heating effect on the coupling of conduction with MHD free convection flow from a vertical flat plate, Nonlinear Analysis: Modeling and Control, Vol.12, No. 3,pp. 307-316.

Merkin J.H and Pop I., (1996), Conjugate free convection on a vertical surface, Int. J. Heat Mass Transfer, Vol. 39, pp.1527- 1534.

Molla M. M, Rahman A. and Rahman T. L., (2005), Natural convection flow from an isothermal sphere with temperature dependent thermal conductivity. J. Architecture and Marine Engineering, Vol.2, pp.53-64.

Keller, H. B., (1978), Numerical methods in boundary layer theory, Annual Rev. Fluid Mech. Vol. 10, pp. 417433. doi:10.1146/annurev.fl.10.010178.002221

Cebeci, T and Bradshaw, P., (1984), Physical and Computational Aspects of Convective Heat Transfer, Springer, New York.

Hossain, M. A. Alim, M. A. and Rees, D. A. S., (1999), The effect of radiation on free convection from a porous vertical plate, Int. J. Heat and Mass Transfer, Vol. 42, pp. 181-191. doi:10.1016/S0017-9310(98)00097-0

Chowdhury, M. K. and Islam, M. N., (2000), MHD Free Convection Flow of Visco-elastic Fluid past an Infinite Porous Plate, Int. J. Heat and Mass Transfer, Vol. 36, No. 5, pp. 439-447. doi:10.1007/s002310000103

Alam, M., Alim, M. A. and Chowdhury, M. M. K., (2007), Free Convection from a vertical permeable circular cone with pressure work and non-uniform surface temperature, Nonlinear Analysis: Modeling and Control, Vol.12, No.1, pp. 21-32.

Miraj, M., Alim, M. A. and Mamun, MAH., (2010), Effect of radiation on natural convection flow on a sphere in presence of heat generation, International Communications in Heat and Mass Transfer, Vol.37, pp. 660-665.

Ali, M. M., Alim, M. A. and Andallah, L. S., (2011), Conjugate effects of Radiation and Joule Heating on Magnetohydrodynamic Free Convection Flow along a sphere with Heat Generation, American Journal of Computational Mathematics, Vol.1, pp. 18-25.

Miraj, M., Alim, M. A., Andallah, L. S., (2011), Effects of pressure work and on natural convection flow around a sphere with heat generation, International Communications in Heat and Mass Transfer, Vol.38, pp. 911-916. 